\title{
The Study of How to Arrange the Asset Structure with the Use of Information Theory and Cybernetics
}

\author{
LIU Baifang ${ }^{1, a}$, CHEN He ${ }^{2, b}$, LIU Xinyu ${ }^{3, c}$ \\ ${ }^{1}$ Business School, Beijing Language and Culture University, Beijing, 100083, China \\ ${ }^{2}$ Business School, Beijing Language and Culture University, Beijing, 100083, China \\ ${ }^{3}$ Economics Research Department,Tokyo University, Tokyo,163-8001, Japan \\ aemail: liubaifang@blcu.edu.cn, bemail:chenhe@blcu.edu.cn, cemail:Ixy200881@126.com
}

Keywords: Asset Structure Optimization;Information Theory; Cybernetics

\begin{abstract}
Information theory and cybernetics pertain to science and technology, but it's theory principle has the role of guiding allocating the enterprise resource at the macro level. From a practical perspective, the assets structure could be regarded as an information system, through the collection, storage and processing of information to realize the control and regulation of the system, thus scheduling the enterprise resources reasonable to make the best use of each asset of the enterprise, avoid wastage and ensure the realization of business objectives.
\end{abstract}

\section{Introduction}

One of the bottlenecks the modern enterprises are facing is the scarcity of available resources, how to allocate the limited resources of the enterprise reasonable and scientific to make the best use of each asset and avoid idle and waste, thus forming a reasonable capital structure should be an important topic of the science of financial management research. In this respect in the 1940s, the information theory and cybernetics which respectively created by the American mathematician Claude Shannon and Norbert has important guiding significance. Asset structure is the proportion of corporate assets of all types to total assets, and its arrangements should ensure the normal operation of the business activities and to obey and serve the overall goal of business finance. The author believes that the assets structure could be regarded as an information system, through the collection, transmission, reception, storage, processing of information and etc. to study the operation of the asset management system, thereby to achieve the control and regulation of the system. This paper will analyze its mechanism of action, in order to inspire the study of the assets structure of the enterprise.

\section{The Basic Principle of the Information Theory, Cybernetics and Their Methodological Significance}

Information theory is created by American mathematician Professor Shannon of Bell Labs, Professor Shannon published two papers -" the mathematical theory of communication " and " communication in the noise " respectively in 1948 and 1949 which marking the generation of information theory. In these two papers, Professor Shannon discusses the problem about information encoding, transmission, decoding and measurement in detail. The central idea of the discussion is that the value of the information is it can eliminate the uncertainty of the objective world, so as to stabilize people's behavior, in the control implement by the controller to which under control, the important aspect is the grasp of the various behavioral states of which under the control, which requires different behavioral information about which under the control. Due to various disturbances, information obtainment will be very difficult, but whether you can get real and reliable information superiority is the key to the implementation of scientific control. On the difficulty of understanding the objective world, there are brilliant expositions of Bertalanffy : “ They are not the object of perception and direct observation, but the conceptual structure. Even the objects in daily life as well, they will never be simply 'given' the sense data or simple 
perception or ...... the difference between real objects and the systems given by observation and 'conceptual' architecture is could not be described with common sense." Therefore, to obtain information about its operational status of objective things is definitely tough task, in the interference of many factors, the way things worked is very complicated.

The principle talked above has profound meaning to the management of corporate assets structure. In the process of arrangement of the corporate assets structure, there needs a profound grasp of the living environment of the enterprise, however, it is often very difficult, because the living environment of the enterprise is not stationary but constantly changing, in addition, the environmental behavior under the stationary state may be very complex. Therefore, managers must deeply understand the importance of information and the difficulty of access to information, only in this way could make the enterprise resource allocation based on a scientific basis, and ultimately enable the enterprises to obtain reasonable and optimal allocation of resources to succeed in the market competition invincible.

Cybernetics is " the infiltrated product of automatic control, electronic technology, radiocommunication, neurophysiology, biology, psychology, medicine, mathematical logic, computer technology, statistical mechanics and other subjects.” From the generation base side of control theory, it is the infiltrated product of multidisciplinary, Wiener, founder of cybernetics is a mathematician, but he was extensively involved in each of these disciplines. The direct motivity of the generation of Cybernetics is the military demands of World War II . Wiener realize the basic principles of automatic anti-aircraft artillery from the hunter's hunting action , namely feedback principle. Feedback principle refers to take the output information of the controlled system again as input information to alter the behavior of the controlled system, for automatic control systems often rely on the transmission mechanism designed within the system to achieve the above purpose, for artificial systems, it is the behavior of the controller that make conscious use of the output information by the controlled system to change the operating state of the system. However, it should be noted that the function condition of the feedback principle is the operation process of the controlled system deviates from the initial setup goals of system operation, that is there are various behavior results of system operation, the randomness and controllability of the operating state is the essential prerequisite of the feedback principle, under the different operate state of system, you should choose a behavior that best to achieve the overall goal of system.

Information theory and cybernetics is inseparable, it is decided by the internal logic that both of them works. About the relationship between the two, the founder of cybernetics Wiener has a brilliant summary: "Control engineering issues and information engineering issues is not able to differentiate, and the key of these issues are not surrounded by electrical technology but the more basic message concept, regardless of the news is transformed by the way of electrical, mechanical or dissemination." Information theory and cybernetics as a scientific methodology is an important guiding ideology of enterprise asset allocation .

The author believes that the allocation of resources is mainly to solve the problem of transmission and obtainment of information, on this basis to make rational arrangement and scientific control of the corporate asset structure, Shannon's information theory made people begin to pay attention to the basic role of information in allocating resources, and access to information is a difficult process, which requires not only scientific and efficient means of information transmission , but high demand to the subject and object themself, the real world is very complex, subject only through scientific means can really realize the essential law of object and make conscious use of these laws in order to achieve the desired results. Although cybernetics comes from the natural sciences, but its basic idea is worth learning by social sciences, if social sciences ignored the new methodology brought about by the other scientific developments, it will miss an excellent development opportunity.

\section{Reasonable Arrangements for Asset Items}

Corporate asset structure itself is a controlled system, it is the business managers use a variety of information to exert purposeful control behavior. Efficient resource allocation behavior need to 
use a lot of advance information to influence the behavior of the various resource allocation, it is because the corrective measures afterwards often cause some waste, sometimes this corrective action does not exist, because of the huge bias may imposes enterprises a higher burden hard to back that towards bankruptcy. This shows that the information, especially prior information is vital important to the system control and the stable development of system, on this issue, Wei Hongsen pointed out after study: " The size of the amount of information of a system that reflects the degree of organization and complexity of the system, it can make the material system to self-regulate in a very economical way. The more complex the system, the more important the message.” Below the author will illustrate this with some of the specific assets items.

Monetary Funds and Short-term Investments

Their main role is to meet the repayment requirements of corporate, the allocation of resources on these projects depends on the company's grasp of their own liabilities, including two aspects: the quantity structures of liabilities and term structure of liabilities. Only accurately master the total amount of corporate debt and the repayment request in different periods, companies can arrange monetary funds and the scale of a variety of short-term investments reasonable, so that highly liquid assets of the enterprises can not only meet repayment requirements but also make less money remain in the low income asset items, thus achieve the best combination of liquidity and profitability of asset structure. It is relatively easy to obtain the information about this, in the management of these items, it can be completed only if there is effective communication between the various corporate sector or positions, in the case of the current rapid development of a variety of financial software, these relevant information has been very easy to obtain, companies can monitor their debt situation at any moments and arrange for the scale of repayment funds reasonable, use the cash floating flow intelligent, minimize the cost of corporate to maintain asset structure liquidity .

\section{Account Receivables}

The size of the accounts receivable depends on the master of the business to their survival environment and their own capability information. This is because accounts receivable is a double-edged sword for business that use properly can expand the size of the company's sales and market share, thus leading to more profit. However, improper use of account receivable will brought the enterprise a higher burden on opportunity cost of capital and a lot of bad debt, thereby reducing the level of corporate total revenue. The author suggests that arranging the scale of account receivables should have information on the following aspects : Market structure, such as current competitive situation of firms and customer's right and position relative to the enterprise; Customer's credit status, such as the customer's solvency, reputation, etc. As well as the enterprise its own business capacity.

From the market structure, if enterprises in a highly competitive industry or customers in a monopoly position and business made a specific investment to meet customer's demand, in order to successfully achieve sales of products and services, corporate may loosen their credit conditions to expand proportion of account receivables , and the opposite situation also exists.

From the customer's credit situation, whether the business-to-customer financing can be take back depends on the customer's repayment ability and their credit rating levels. Financial theorists believe that the measure of a customer solvency standards generally the "5C" system, but these standards generally have static nature which can not reflect the dynamic characteristics of corporate solvency, therefore we should maintain appropriate trust and appropriate vigilance for these solvency standards. In the developed market economies, there is a lot of the credit rating agencies that publish the credit level of each public companies regularly, although they has advantages in terms of credit rating as a professional organization, but the corporate scandals appears in United States and Europe in recent years tells us in the case that information can not be completely symmetrical, the corporate should keep credit scale from any customer to avoid significant impact on their operations due to unexpected bankruptcy of large customers, meanwhile enterprises should seek other sources to know whether the customer's operation is normal and the change of their solvency. 
In addition, the company's own operate ability will influence the scale of account receivables, objectively speaking, different companies have their own unique ability that determine their competitive advantage in the marketplace, companies should objectively assess their management capabilities. It must be admitted that due to various constraints, such as the quality of personnel, this ability distributed unevenly among the enterprises, which will exert a certain influence on the allocation of corporate financial resources, companies must based on the fully aware of their operate ability to formulate financial policies and strengthen management of accounts receivable .

Inventory

Inventory is an important current assets of enterprise, but from the perspective of corporate value creation, it is a waste that funds occupied in the inventory. Business inventory can be divided into two parts: one is various reserves for the production; the other is new value that created. The optimal goal of enterprise inventory control is: various reserves supplied for the production can be carried out immediately and new value created can be sale immediately. The information that enterprises need for inventory control involves a number of aspects, including market structure, production and operation characteristics of enterprise and so on.

In addition to these information, the effective transfer of information between enterprises and customers as well as suppliers is vital important to inventory management, which involves technical issues of information transmission in information theory. Recently, the development of network technology which greatly improves the efficiency of information transmission between the enterprises which its industrial chain performance vertically, some enterprises take advantage of the latest developments in information technology to control the efficiency of resource allocation in their inventory effectively. The company which achieved optimal inventory control by use of information technology should be the U.S. computer company - DELL company. The company make use of its own website to transfer their own needs plans for the various raw materials to various suppliers immediately, and achieved the order production by use of direct selling, its corporate headquarter take advantage of information technology to adjusted its production plan once every two hours over the world in order to achieve rational use of resources in the world, in this inventory control that the inventory level could meet only three to four days' customer needs, thus greatly reducing the occupy of inventory to scarce resources and improving the efficiency of resource utilization.

Intangible Assets

Intangible assets have become a key factor in gaining access to sustainable competitive advantage and excess profits, such as various brands, patents, non-patented technology and so on. It should be noted that the obtainment of those rights are not without cost, it is also the results of resources input. There are many factors to consider whether the enterprises to develop an intangible asset, I think the time and environment, industry characteristics, market structure and target customers are all important factors and can be summarized as environmental information .

\section{Conclusion}

According to Shannon information theory, there is active or passive access to information. For enterprises, passive perception is only felt when changes in the external environment will threaten its survival mode; while active perception is the enterprise act as cognitive subject to observing small changes of the living environment of the enterprise initiatively and made a deep understanding of the impact that these changes on the production and operation of enterprises, so that promote the beneficial and abolish the harmful, then avoid misunderstandings and prevent crises before they emerge. Since the establishment of the market economy in China is not long, many companies do not establish the appropriate marketing values and sense of competition. Particularly, the inadequate attention to intangible assets, poor brand awareness and less fully awareness of the fundamental role of knowledge assets to business growth .

From a realistic point of view, multinational companies have become our major competitors, these companies thoroughly tempered in international competition that cultivate a unique competitive capabilities and a strong competitive advantage, if enterprises of China want to 
compete with them , we must establish the advanced management philosophy, change the resource allocation behavior timely, cultivate the unique resources and core competitiveness of enterprises, build long-term competitive advantage.

In summary, information theory and cybernetics as technology basic science could guide the allocation of resources from the macro level and arrange for asset structure reasonable. As a modern enterprise, smooth financial information transmission system and feedback system must be built based on the need of business operate strategy, then managers could understand the situation beforehand, strengthen control, manage the enterprise resources rationally, improve operational efficiency of assets. And through the information feedback to adjust related instructions timely, reduce business risk and ensure the successful completion of corporate business objectives.

\section{References}

[1] Titman S.. The effect of capital structure on a firm's liquidation decision [J]. Journal of Financial Economics, 1984,13,137-151.

[2] Brander J. and Lewis T. Oligopoly and financial structure: the limited liability effect[J]. American Economic Review, 1986, 76, 956-970.

[3] Bolton P. and D. Scharfstein. A Theory of predation based on agency problems in financial contracting[J]. American Economic Review, 1990, 80, 93-106.

[4] Maksimovic V. and Titman S. Financial reputation and reputation for product quality[J], Review of Financial Studies, 1991, 2, 175-200.

[5] Masulis R. Stock repurchase by tender offer, an analysis of the cause of common stock price changes[J]. Journal of Finance,1980, 35. 